\title{
Concurrent Chemoradiation
}

National Cancer Institute

\section{Source}

National Cancer Institute. Concurrent Chemoradiation. NCI Thesaurus. Code C141342.

Treatment in which radiation therapy is administered at the same time as chemotherapy. 